\title{
INHALTSVERZEICHNISS.
}

ERSTES BUCH.

I. DIE AUFGABE . . . . . . . . . . . . . . . . 3

II. DIF LAGE . . . . . . . . . . . . . . . . . 12

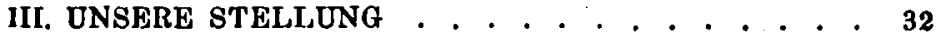

IV. DIE CONSERVATIVEN . . . . . . . . . . . . 37

ZWEITES BUCH.

I. DER SCHWERPUNKT . . . . . . . . . . . . . 47

II. DER ECHTE HORAZ . . . . . . . . . . . . . 61

DRITTES BUCH.

I. GEGNER . . . . . . . . . . . . . . . . . . . . . 69

II. BUNDESGENOSSEN . . . . . . . . . . . . . . . 76

III. EIN NEUER VERSUCH

IV. DER HORAZ VON LEHRS ........... 83

VIERTES BUCH.

I. DIE ODEN . . . . . . . . . . . . . . . . . . . . . . . . . 91

II. LEHRS GANZE BESTREBUNG . . . . . . . . . 117

FUENFTES BUCH.

I. DIE LUECKEN . . . . . . . . . . . . . . 125

II. DIE UMSTELLUNG . . . . . . . . . . . . . . 132

SECHSTES BUCH.

I. DIE SOLIDEN . . . . . . . . . . . . . 139

II. BENTLEYS PHAEDROS . . . . . . . . . . . . 153

III. DAS EPICEDIUM DRUSI . . . . . . . . . . . . 157

IV. EIN ZWISCHENFALL . . . . . . . . . . . . . . . . 166

SIEBENTES BUCH.

I. NEUER GROND . . . . . . . . . . . . . . . . 175

II. AEUSSERE BEWEISMITTEL . . . . . . . . . . 182

III. ARTEN DER FAELSCHONG . . . . . . . . . . . 184

IV. ZEIT DER FAELSCHUNG . . . . . . . . 188 
V. UMFANG DER AUSSCHEIDUNGEN . . . . . . 196 VI. UEBERBLICK . . . . . . . . . . . . . . . 199

ACHTES BUCH.

NACHTRAEGE

NEUNTES BUCH.

\begin{tabular}{|c|c|c|c|c|c|c|c|c|c|c|c|c|c|c|c|c|}
\hline I. & & I, 2 & $\cdot$ & $\cdot$ & & • & $\cdot$ & $\cdot$ & & & & $\bullet$ & & •" & & \\
\hline II. & ", & I, 3 & 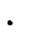 & . & $\cdot$ & . & . & . & . & & & . & . & . & & 227 \\
\hline III. & ", & I, 4. & . & 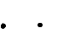 & 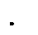 & 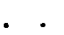 & 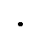 & . & & & & & . & . & & \\
\hline IV. & $"$ & I, 5 & . & . . & . & . & $\cdot$ & . & 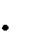 & & . & 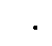 & . & . & & \\
\hline V. & ", & $I, 6$. & - & . & . & - & $\cdot$ & · & . & & . & . & . & . & & \\
\hline VI. & $"$ & $\mathrm{I}, 7$. & . & . & . & . & · & . & . & & & • & . & . & & \\
\hline VIL & $"$ & I, 10 & . & - & . & 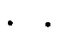 & . & . & . & 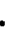 & - & - . & . & . & & \\
\hline III. & ", & II, 3 & . & . & & & , & & & & . & . . & - & . & & \\
\hline IX. & ," & II, 8 & . & - & & & 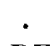 & & & . & 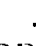 &. & . & . & & \\
\hline
\end{tabular}

ZEHNTES BUCH.

I. EPIST. I, 1 . . . . . . . . . . . . . . . . . 273

II.,$\quad$ I, 2. . . . . . . . . . . . . . . . 277

III. $\quad, \quad$ I, 5. . . . . . . . . . . . . . . . . . 281

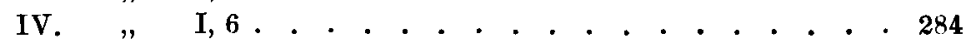

V. " I, 7. . . . . . . . . . . . . . . . . 288

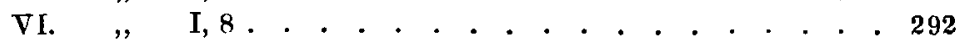

VII. $" \quad$ I, 11 . . . . . . . . . . . . . . . . . 294

VIII. $" \quad \mathrm{I}, 12$. . . . . . . . . . . . . . . 297

IX. $, \quad \mathrm{I}, 13$. . . . . . . . . . . . . . . . 300

X. " I, 15 . . . . . . . . . . . . . . . . 305

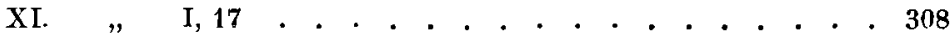

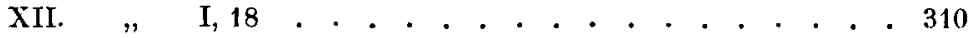

XIII. " I, 19 . . . . . . . . . . . . . . . . . 315

XIV. " II, 1 . . . . . . . . . . . . . . . . 320

XV. $\quad$ II, 2 . . . . . . . . . . . . . . . . . 331

XVI. DER BRIEF AN DIE PISONEN . . . . . . . . . 334

ELFTES BUCH.

I. EPODUS I . . . . . . . . . . . . . . 349

II. EPOD. VI . . . . . . . . . . . . . . 352

III. " XVI . . . . . . . . . . . . . . . . . 354

IV. $"$ IX . . . . . . . . . . . . . 356

V. " XII, X, XIII . . . . . . . . . . . 361

VI. $"$ VIII, XI, II . . . . . . . . . . . . . . 364

VII. " IX, XV . . . . . . . . . . . . . . . 368

VIII. " II . . . . . . . . . . . . . . . 371 


\section{ZWOELFTES BUCH.}

Beito

I. ODE II, 6 . . . . . . . . . . . . . . . 377

II. " I, 7 . . . . . . . . . . . . . . 384

III. " III, 17 . . . . .......... . . . 389

IV. " I, 37 . . . . . . . . . . . . . . . . 393

V. "II, 2. . . . . . . . . . . . . . . . . . 401

VI. DIE MALERISCHEN SCHLUESSE . . . . . . . . 404

VII. ODE I, 12 . . . . . . . . . . . . . . . . . 407

VIII. " III, 21; I, 9; I, 14............. . 411

IX. " IV, 9................ . 416

X. " II, 16 . . . . . . . . . . . . . . . . . 418

XI. " III, 27 . . . . . . . . . . . . . . . . . 423

XII. " III, 5. . . . . . . . . . . . . . . . . . 427

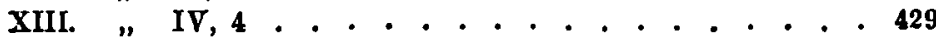

XIV. " III, 16 . . . . . . . . . . . . . . . . . 433

XV. " III, 11 . . . . . . . . . . . . . . . . . 437

XVI. " IV, 2 . . . . . . . . . . . . . . . . . 441

XVII. " III, 7 . . . . . . . . . . . . . . . . . 445

XVIII. " III, 20 . . . . . . . . . . . . . . . . . 446

XIX. " III, 1, 2, 3 . . . . . . . . . . . . . . . 449

XX. " III, 4 . . . . . . . . . . . . . . . . . 456

XXI. " I, 34, 35 . . . . . . . . . . . . . . . . 460

DREIZEHNTES BUCH.

I. DIE KURZEN STUECKE. . . . . . . . . . . . 465

II. ODE III, 29 . . . , . . . . . . . . . . . . . 467

III. " II, 17 . . . . . . . . . . . . . . . . . 470

IV. ., III, 26; $1 \mathrm{~V}, 1$. . . . . . . . . . . . . . 473

V. " I, 33; I, 19 . . . . . . . . . . . . . . . 475

VI. " I, 36.... . . . . . . . . . . . . 477

VII. " 1,$20 ; 1,30$. . . . . . . . . . . . . . 479

VIII. " IV, 13 . . . . . . . . . . . . . . . 481

IX. " II, 5; I, 23 . . . . . . . . . . . . . . . . 483

X. " II, 18 . . . . . . . . . . . . . . 486

XI. " IV, 8................ . 490

XII. "IV, 7. . . . . . . . . . . . . . . . . 494

XIII. " DIE DUERF'IIGEN STUECKE . . . . . . 497

XIV. " I, 17 . . . . . . . . . . . . . . . . 504

XV. " III, 13 . . . . . . . . . . . . . . . 507

XVI. " I, 8 . . . . . . . . . . . . . . . . . . 509

XVII. " III, 12 . . . . . . . . . . . . . . . . . 512

XVIII. DIE SCHLUSSGEDICHTE . . . . . . . . . 514

VIERZEHNTES BUCH.

I. LALAGE . . . . . . . . . . . . . . . . 520

II. LYDIA . . . . . . . . . . . . . . . . 524 
III. DIE EROTISCHEN ODEN . . . . . . . . . . . 531

IV. DIE EROTISCHEN MOTIVE . . . . . . . . . . 534

FUNFZEHNTES BUCH.

I. DIOMEDES UND MARIUS VIC'IORINUS . . . . . . 542

II. METRISCHE ARGUMEN'TE . . . . . . . . . . . 549

III. VERHAELTNISS ZU DEN GRIECHEN . . . . . . 559

IV. ART DER ANKNUEPFUNG . . . . . . . . . . . 5664

V. WETTEIFERNDE INTERPOLATIONEN . . . . . . 568

VI. DIE VIERZEILIGEN STROPHEN . . . . . . . . 583

VII. STELLUNG DES UNECHTEN . . . . . . . . . . . 589

VIII. DIE, BUECEERTHEILUNG . . . . . . . . . . . 593

IX. DIE ANORDNUNG . . . . . . . . . . . . . . . 597

X. DAS CHRONOLOGISCHE . . . . . . . . . . . . 601 SCHLUSSWOR'l'. . . . . . . . . . . . . . . . 607 ZUSAE'TZE UND BERICHTIGUNGEN . . . . . . . 612 VERZEICHNISS DER BEHANDEL'TEN 'TEX'I'S'TELLEN 614 